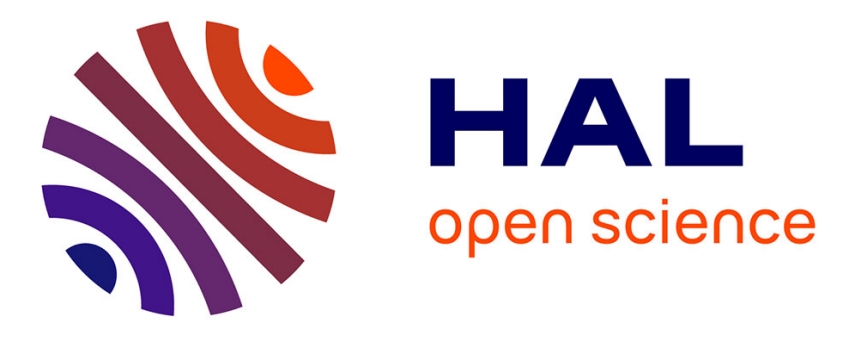

\title{
Probing ultrafast thermalization with field-free molecular alignment
}

\author{
Julien Houzet, Julien Gateau, Edouard Hertz, Franck Billard, Bruno Lavorel, \\ Jean-Michel Hartmann, Christian Boulet, Olivier Faucher
}

\section{- To cite this version:}

Julien Houzet, Julien Gateau, Edouard Hertz, Franck Billard, Bruno Lavorel, et al.. Probing ultrafast thermalization with field-free molecular alignment. Physical Review A : Atomic, molecular, and optical physics [1990-2015], 2012, 86, pp.033419. 10.1103/PhysRevA.86.033419 . hal-00750053

\section{HAL Id: hal-00750053 https://hal.science/hal-00750053}

Submitted on 7 Dec 2012

HAL is a multi-disciplinary open access archive for the deposit and dissemination of scientific research documents, whether they are published or not. The documents may come from teaching and research institutions in France or abroad, or from public or private research centers.
L'archive ouverte pluridisciplinaire HAL, est destinée au dépôt et à la diffusion de documents scientifiques de niveau recherche, publiés ou non, émanant des établissements d'enseignement et de recherche français ou étrangers, des laboratoires publics ou privés. 


\title{
Probing ultrafast thermalization with field-free molecular alignment
}

\author{
J. Houzet ${ }^{1}$, J. Gateau ${ }^{1}$, E. Hertz ${ }^{1}$, F. Billard ${ }^{1}$, B. Lavorel ${ }^{1}$, J.-M. Hartmann ${ }^{2}$, C. Boulet ${ }^{3}$, and O. Faucher ${ }^{1}$ \\ (1) Laboratoire Interdisciplinaire Carnot de Bourgogne (ICB), UMR 6303 CNRS-Université de Bourgogne, \\ 9 Av. A. Savary, BP 47 870, F-21078 DIJON Cedex, France \\ (2) Laboratoire Interuniversitaire des Systèmes Atmosphériques (LISA), \\ UMR 7583 CNRS-Université Paris Est Créteil, Université Paris Diderot, \\ Institut Pierre-Simon Laplace, 94010 Créteil Cedex, France and \\ (3) Institut des Sciences Moléculaires d'Orsay (ISMO), \\ UMR 8214 CNRS-Université Paris-Sud, Bât. 350, Orsay F-91405, France
}

(Dated: September 3, 2012)

\begin{abstract}
The rotation-translation thermalization of $\mathrm{CO}_{2}$ gas is investigated $500 \mathrm{ps}$ after its preheating by a non-resonant short and intense laser pulse. The temperature of thermalization is optically determined with two additional short laser pulses enabling a field-free molecular alignment process and its probing, respectively. The measurements are performed for various intensities of the preheat pulse leading to the observation of different temperatures which are in very good agreement with classical molecular dynamics simulations. The results can be regarded as a step towards real time tracking of ultrafast relaxation pathways in molecular motion.
\end{abstract}

PACS numbers: $37.10 . \mathrm{Vz}, 34.50 . \mathrm{Ez}, 42.50 . \mathrm{Md}$

\section{INTRODUCTION}

With the advent of short and intense laser pulse technology routinely available in laboratories, light-induced molecular alignment has become a well-established method for controlling the spinning of a molecule about a privileged direction. The basics have been explored through a wealth of experimental $[1,2]$ and theoretical [3] studies. The effect is particularly interesting when produced with a non-resonant pulse of duration much shorter than the classical rotational period of the molecule. In this regime, the interaction leads to transiently aligned molecules produced after extinction of the driving field. This so-called field-free alignment is appropriate to a growing class of potential applications including molecular tomography [4], molecular-frame photoelectron angular distribution [5], laser filamentation [6], control of molecular scattering [7], high-order harmonic generation [8], and collisional dynamics of liquid and gas phase systems [9]. The present work is related to the latter topic.

The benefit of using molecular alignment for probing dissipative environments was addressed by the pioneer work of Ramakrishna et al. [10]. The temporal evolution of field-free alignment is featured by transient components, during which the molecules are either delocalized in a plane or aligned along a fixed axis, together with a steady state component corresponding to a permanent alignment $[1,2]$. The dynamics is more or less complex depending on the molecular symmetry. Considering the simplest case of linear molecules like $\mathrm{CO}_{2}$ used in the present study, the transients revive at a fixed time interval corresponding to a fraction of the rotational period. Dissipation due to collisions differently affects the transients and permanent component of the alignment. A reason is that the former arises from optical coherences whereas the latter occurs due to populations, both ro- tationally excited during the pulse interaction through stepwise Raman transitions [11]. Revivals coincide with the rephasing of rotational coherences that is sensitive to both state-changing ( $J$ and $M$ ) and -dephasing collisions, while populations are only affected by the former. Revivals and permanent alignment therefore decay with different rates due to pure dephasing processes [10]. This difference is moreover considerably enhanced considering that collisions (at least for $\mathrm{CO}_{2}$ ) have a propensity to conserve the orientation of the angular momentum (i.e., $M / J)[12]$. This last mechanism and its important consequences for the decays of the permanent and transient alignments are supported by recent measurements performed on aligned $\mathrm{CO}_{2}$ molecules in pure gas phase and in $\mathrm{CO}_{2}-\mathrm{He}$ gas mixtures [13].

\section{METHODS}

Relaxation processes in molecular dynamics are governed by the energy transfers occurring among the different degrees of freedom of motion. For instance, extensive literature can be found about vibrational relaxation processes studied with photoacoustic spectroscopy $[14,15]$. This technique is well adapted to measure the relaxation due to vibration-translation (V-T) transfers which typically occurs at the microsecond time-scale for $\mathrm{CO}_{2}$ molecules at atmospheric pressure. [16]. However, it is not relevant to much faster relaxations involving rotational and translational motions. The present study demonstrates that rotational-translation $(\mathrm{R}-\mathrm{T})$ transfers can be optically probed in the few hundreds of picosecond time-scale using the concept of field-free molecular alignment.

The alignment of a linear molecule is frequently described in terms of $\left\langle\cos ^{2} \theta\right\rangle$, where $\langle\cdot\rangle$ refers to the quantum expectation value and $\theta$ is the angle of the molecular 
axis with respect to the field polarization. A molecule aligned along the field is achieved when $\left\langle\cos ^{2} \theta\right\rangle>1 / 3$, whereas $\left\langle\cos ^{2} \theta\right\rangle<1 / 3$ reflects a delocalization of the molecular axis in the plane perpendicular to the field, $1 / 3$ being the degree of alignment representing a randomly oriented molecule. To describe a gas sample, this quantity must be averaged over the initial states $(J, M)$ of the constitutive molecules, which is properly described at thermal equilibrium by the Maxwell-Boltzman statistics: ${\overline{\left\langle\cos ^{2} \theta\right\rangle}}_{T}(t)=\sum_{J} \sum_{M=-J}^{M=J} \rho_{J}\left\langle\cos ^{2} \theta\right\rangle_{J, M}$. In this expression, $T$ is the rotational temperature of the gas sample before interaction, $J$ and $M$ are the total angular momentum and its projection along the quantification axis, respectively, and $\rho_{J}=\frac{g_{J} \exp \left(-E_{J} / k_{\mathrm{B}} T\right)}{\sum_{J}(2 J+1) g_{J} \exp \left(-E_{J} / k_{\mathrm{B}} T\right)}$ is the thermal relative population with $k_{\mathrm{B}}$ the Boltzmann constant, $E_{J}$ the rotational energy, and $g_{J}$ the nuclear spin degeneracy factor to be considered only in case of symmetric molecules. Due to the thermal averaging, a change in the temperature and therefore in the initial state distribution affects both the amplitude and shape of $\overline{\left\langle\cos ^{2} \theta\right\rangle_{T}}(t)$. This dependence with respect to $T$ is a feature that makes the concept of field-free alignment particularly useful for non-resonant space-resolved optical diagnostics $[17,18]$.

\section{RESULTS AND DISCUSSION}

The experiment is performed in $\mathrm{CO}_{2}$ at a static pressure of 1 bar and initially at $295 \mathrm{~K}$. The alignment produced by a strong pulse is optically probed by a weak pulse using a time-resolved birefringence technique and an homodyne detection. As shown previously [19, 20], the technique provides a signal $\mathcal{S}$ proportional to the temporal convolution of ${\overline{\left(\left\langle\cos ^{2} \theta\right\rangle-1 / 3\right)_{T}}}_{T}^{2}(t)$ with the temporal envelope of the probe pulse. The aligning and probing pulses are produced by a chirped pulse amplified Ti:sapphire femtosecond laser which delivers $100 \mathrm{fs}-$ duration pulses at a repetition rate of $100 \mathrm{~Hz}$, with a wavelength centered at $800 \mathrm{~nm}$. The signal of the first alignment revival recorded at room temperature (solid line) is shown in Fig. 1(a). The intensity of the aligning pulse is set to $25 \mathrm{TW} / \mathrm{cm}^{2}$ and the alignment produced by the weak probe can be neglected. The other revivals spaced by $T_{\mathrm{r}} / 4$, with $T_{\mathrm{r}}$ the rotational period [19], are not shown. The corresponding first revival signal obtained when introducing a laser pulse of $60 \mathrm{TW} / \mathrm{cm}^{2} 500$ ps prior to the aligning pulse is shown on the same figure. This preliminary pulse serves as a preheat pulse. A part of its energy is transferred to the $\mathrm{CO}_{2}$ molecules rotations by means of intrapulse non-resonant Raman excitations of the rotational levels. It is then converted to translation through R-T transfers until complete thermalization (full Boltzmann equilibrium of both rotation and translation) is reached. Figure 2 displays the predicted temporal evolutions of the averaged rotational and translational energies following the excitation of the system by
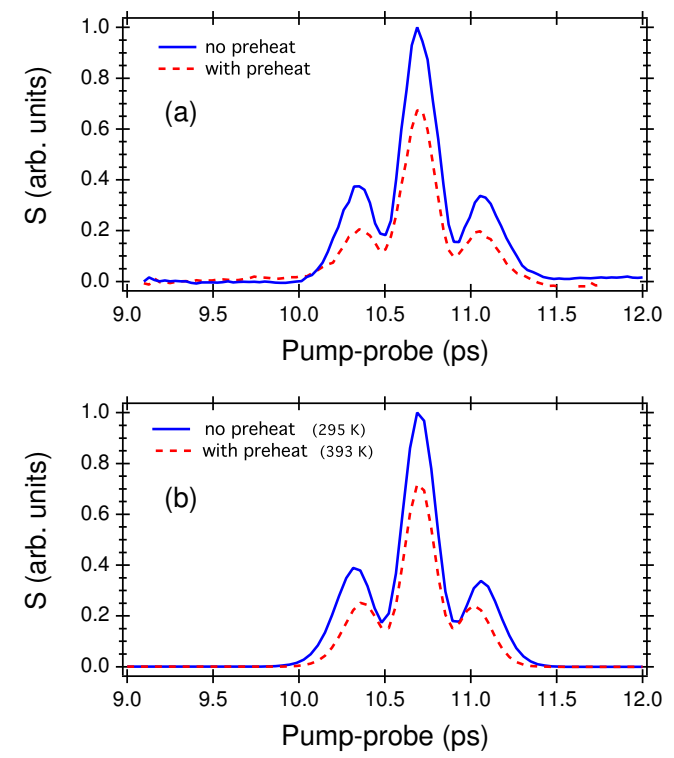

FIG. 1: (Color online) (a) Alignment induced birefringence measured with (dashed red line) and without (solid blue line) preheating a $\mathrm{CO}_{2}$ gas sample by a short laser pulse launched 500 ps before initiating the alignment. (b) Alignment signal versus time calculated with the quantum-ECS model for a rotational temperature computed by CMDS (see text).

the preheat pulse. These were obtained from Classical Molecular Dynamics Simulations (CMDS) as described in [12] and using the $\mathrm{CO}_{2}-\mathrm{CO}_{2}$ intermolecular potential of [21]. Immediately after the preheat pulse, set at the time origin, the average energy released by the field into the rotation raises to about $E_{\mathrm{rot}} / k_{\mathrm{B}} \approx 520 \mathrm{~K}$. Then collisions induce changes leading to the decrease of the rotational energy and increase of the translational one with a progressive equilibrium within each of these modes until they are in equilibrium with each other. According to CMDS, Rotation-Rotation (R-R) relaxation is faster than $\mathrm{R}-\mathrm{T}$ relaxation, with respective time constants of $70 \mathrm{ps}$ and $150 \mathrm{ps}$ for pure $\mathrm{CO}_{2}$ at $1 \mathrm{bar}$, and an average time between collisions of 100 ps. The results plotted in Fig. 2 together with Fig. 15 of [12] thus predict that, after typically $500 \mathrm{ps}$, a Boltzmann equilibrium is reached within rotation and translation, these two degrees of freedom being (almost) in equilibrium with each other, i.e., $E_{\text {rot }} \approx 2 / 3 E_{\text {trans. }}$. The modifications of the alignment revival observed in Fig. 1(a) reflects hence the elevation of the (equilibrium) rotational temperature produced after thermalization of the gas sample. It is worth mentioning that aligning and preheat pulses have been exchanged to make sure that the pulses were not affected by thermal effects in the optics. Propagation effects due to the plasma generated by the preheat pulse have also been tested by performing the experiment in Argon, observing the electronic Kerr response of the gas. In this case, no signal change introduced by the preheat pulse could be noticed. 


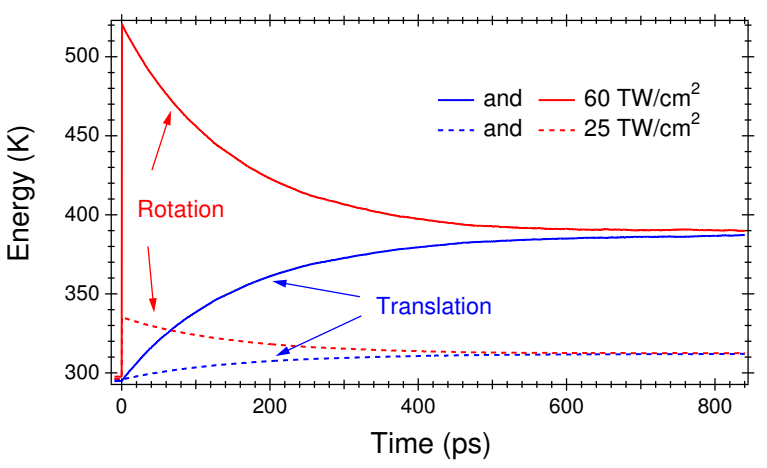

FIG. 2: (Color online) Averaged rotational energy $E_{\text {rot }} / k_{\mathrm{B}}$ (red curves) and translational energy $2 E_{\text {trans }} / 3 k_{\mathrm{B}}$ (blue curves) of $\mathrm{CO}_{2}$ molecules initially excited by a 100 fs-duration laser pulse of $60 \mathrm{TW} / \mathrm{cm}^{2}$ (solid lines) and $25 \mathrm{TW} / \mathrm{cm}^{2}$ (dashed lines). The initial temperature of the gas sample is $295 \mathrm{~K}$ and the pressure is $1 \mathrm{bar}$.

To confirm the observed effect, we performed simulations of the birefringence signal on the first alignment revival of $\mathrm{CO}_{2}$. For this, the rotational temperature $500 \mathrm{ps}$ after the preheat pulse was first calculated using CMDS (e.g., Fig. 2). The expectation value $\left\langle\cos ^{2} \theta\right\rangle$ following the aligning pulse was then calculated using the quantum M-dependent Energy Corrected Sudden (ECS) model of [12]. The previously used values $[12,13]$ of the ECS parameters $\left(A, \alpha, \beta, \ell_{\mathrm{c}}\right)$ were retained with the multiplication of $A$ (in ps/atm units) by $(295 / T)^{0.95}$ in order to accurately represent the temperature dependent self broadening values of [22]. These quantum-ECS calculations thus provide the values of $\left\langle\cos ^{2} \theta\right\rangle$ following a 25 $\mathrm{TW} / \mathrm{cm}^{2}$ aligning pulse for rotational temperatures associated, through CMDS, with preheat pulses of different intensities. Note that [12] shows that the classical and quantum approaches lead to consistent predictions for the collisional dissipation of laser induced alignment confirmed in [13] by comparisons with experimental results. The simulations conducted with (solid line) and without (dashed line) preheat pulse are shown in Fig. 1(b). Both results agree with the observations reported in Fig. 1(a). This comparison allows to estimate the rotational temperature $(\approx 400 \mathrm{~K})$ resulting from preheating the gas sample by the $60 \mathrm{TW} / \mathrm{cm}^{2}$ laser pulse. To our knowledge, this is the first measurement of temperature increment resulting from ultra-fast R-T transfers. We point out that the calculations of Fig. 1(b) were performed at the same molecular density, a safe assumption considering the value of the delay between the preheat and aligning pulses. Indeed, the average distance traveled by $\mathrm{CO}_{2}$ molecules at $400 \mathrm{~K}$ during $500 \mathrm{ps}$ is less than $0.3 \mu \mathrm{m}$, a value negligible when compared to the beam foci $(\approx 30 \mu \mathrm{m})$. Note that the rotational thermalization of the gas sample has also been confirmed by observing the extinction of the field-free alignment produced by the preheat pulse after 500 ps. No residual permanent nor

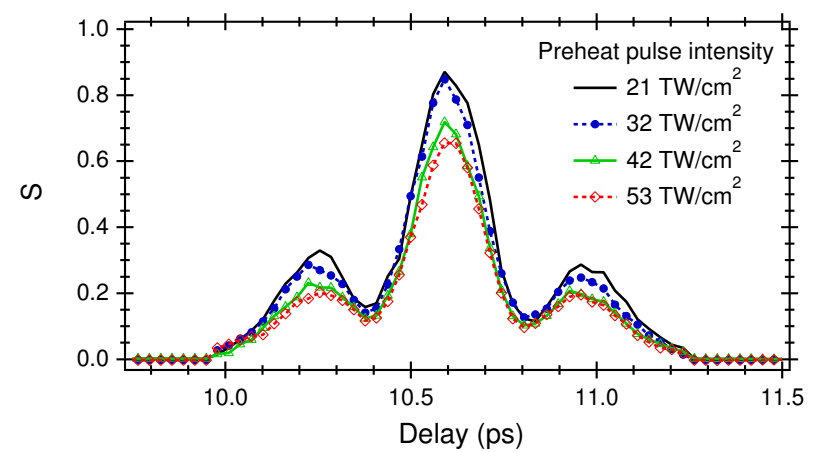

FIG. 3: (Color online) First alignment revival of $\mathrm{CO}_{2}$ recorded at different intensities of the preheat pulse.

transient alignment was observed indicating that a Boltzmann equilibrium is reached within rotational degrees of freedom.

In a first approximation (valid for moderate pulse intensities), the internal energy of the molecules increases with the square of the applied intensity (e.g., Fig. 6 of [12]). This is due to the rotational excitations that are achieved through Stimulated Raman-like transitions in an intensity regime well described by two-photon processes. As already mentioned, the rotational temperature of the gas sample can be deduced from the modification of the alignment signal. The $\mathrm{CO}_{2}$ signal recorded for different intensities of the preheat pulse is shown in Fig. ?? Figure 4 presents a comparison between the total area of the alignment revival recorded for different intensities and the theoretical predictions from the quantumECS calculations carried for the rotational temperature derived from CMDS. The values are normalized to the area obtained without preheat pulse. As can be seen the agreement between experimental and calculated values is very good. This is a noticeable success for simulations free of any adjusted parameter that, together with the results of [13], confirms the quality of the approaches proposed in [12]. The right hand scale of Fig. 4 provides the equilibrium temperature of the molecules after complete thermalization.

The elevation of temperature resulting from the preheat pulse also influences the long-term evolution of the alignment signal through a decay modification. This has been confirmed by measuring at 1 bar the area of the first and fifth transients (separated by $42.7 \mathrm{ps)} \mathrm{with} \mathrm{and} \mathrm{with-}$ out a $60 \mathrm{TW} / \mathrm{cm}^{2}$ preheat pulse. The exponential fit of the data collected over several days indicates a time decay of 55.2 ps with preheat, against 59 ps without preheat, with a corresponding ratio of $0.937 \pm 0.063$. These values are in agreement with the CMDS [12] predictions, i.e., 54.2 and $59.5 \mathrm{ps}$, respectively. The faster decay (6.4\%) observed with the preheat pulse is consistent with the increase of temperature detected just after thermalization, i.e., before variation of the molecule density in the interaction volume. 


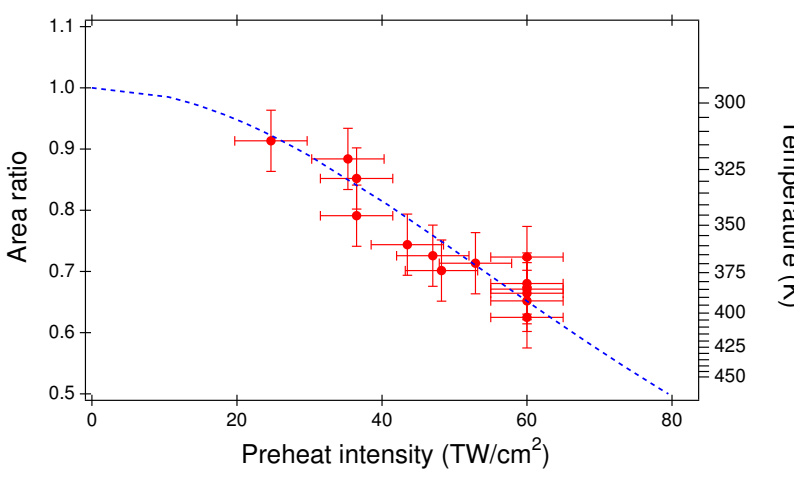

FIG. 4: (Color online) Area of the first alignment revival normalized to the value measured with no preheat pulse: experiment (red solid circles) and theory (blue dashed line).

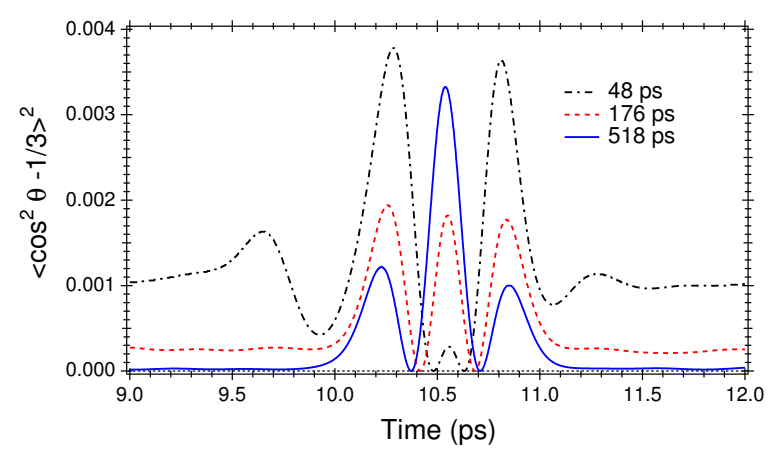

FIG. 5: (Color online) First alignment revival of $\mathrm{CO}_{2}$ calculated for different temporal delays between the preheat and alignment pulse.

\section{CONCLUSION}

Field free molecular alignment has been used to optically probe the ultrafast temperature rising in a molec- ular sample subsequent to the increase of its rotational energy by a short and intense laser pulse. The detection has been conducted by setting the delay between this preheat pulse and the detection pulses early after thermalization in order to limit the migration of molecules out of the laser foci during the measurements. By varying the intensity of the preheat pulse, we have been able to detect an incremental temperature from nearly zero up to about $100 \mathrm{~K}$ above the initial temperature (295 $\mathrm{K}$ ) in good agreement with the predictions of classical molecular dynamics simulations. A similar heating effect resulting from $\mathrm{R}-\mathrm{T}$ transfers after laser excitation is expected in the production of the laser induced gas vortices predicted very recently [23]. As an extension of the work, a stimulating perspective would be to perform the optical diagnostics for various delays between excitation and thermalization of the sample. For a preliminary investigation of the information brought by such experiments, CMDS have been made for a preheat pulse of $60 \mathrm{TW} / \mathrm{cm}^{2}$ and a $20 \mathrm{TW} / \mathrm{cm}^{2}$ alignment pulse. The subsequent first alignment revivals obtained for various delays between these two pulses are plotted in Fig. 5 . They show a considerable increase of the central peak near 10.5 ps when increasing the delay together with a simultaneous decrease of the two surrounding peaks for the chosen time delays. Adapted experiments would enable to track these evolutions, providing an additional stringent test of the theoretical models and the possibility to analyse the influence of $\mathrm{R}-\mathrm{R}$ and $\mathrm{R}-\mathrm{T}$ relaxations that the evolution of the revival shape translates. Such experiments may enable the first direct measurement of $\mathrm{R}-\mathrm{T}$ transfer rates through investigations at the relevant time scale. This seems a promising alternative to the very indirect extraction of this rate and of "rotational collision numbers" from bulk viscosity or thermal transpiration measurements (eg [24] and those cited in Fig. 2 of $[25])$.
[1] H. Stapelfeldt and T. Seideman, Rev. Mod. Phys. 75, 543 (2003).

[2] V. Kumarappan, S. S. Viftrup, L. Holmegaard, C. Z. Bisgaard, and H. Stapelfeldt, Phys. Scr. 76, C63 (2007).

[3] T. Seideman and E. Hamilton, Adv. At. Mol. Opt. Phys. 52, 289 (2006).

[4] M. Meckel, D. Comtois, D. Zeidler, A. Staudte, D. Pavičić, H. C. Bandulet, H. Pépin, J. C. Kieffer, R. Dörner, D. M. Villeneuve, et al., Science 320, 1478 (2008).

[5] J. L. Hansen, H. Stapelfeldt, D. Dimitrovski, M. Abusamha, C. P. J. Martiny, and L. B. Madsen, Phys. Rev. Lett. 106, 073001 (2011).

[6] F. Calegari, C. Vozzi, and S. Stagira, Phys. Rev. A 79, 023827 (2009).

[7] E. Gershnabel and I. S. Averbukh, Phys. Rev. A 82, 033401 (2010).
[8] S. Haessler, J. Caillat, W. Boutu, C. Giovanetti-Teixeira, T. Ruchon, T. Auguste, Z. Diveki, P. Breger, A. Maquet, B. Carré, et al., Nature Phys. 6, 200 (2010).

[9] S. Ramakrishna and T. Seideman, J. Chem. Phys. 124, 034101 (2006).

[10] S. Ramakrishna and T. Seideman, Phys. Rev. Lett. 95, 113001 (2005).

[11] T. Vieillard, F. Chaussard, D. Sugny, B. Lavorel, and O. Faucher, Journal of Raman Spectroscopy 39, 694 (2008).

[12] J.-M. Hartmann and C. Boulet, J. Chem. Phys. 136, $184302(2012)$.

[13] T. Vieillard, F. Chaussard, F. Billard, D. Sugny, S. Yvanov, J.-M. Hartmann, C. Boulet, O. Faucher, and B. Lavorel (to be submitted).

[14] J. Lambert, Vibrational and Rotational Relaxation in Gases. (Clarendon, Oxford, 1977). 
[15] A. Tramer, C. Jungen, and F. Lahmani, Energy dissipation in molecular systems. (Springer, Berlin, 2005).

[16] F. Cannemeijer and A. E. De Vries, Physica 64, 123 (1973), ibid 70, 135 (1973).

[17] H. Tran, B. Lavorel, O. Faucher, R. Saint Loup, and P. Joubert, J. Raman Spectrosc. 34, 994 (2003).

[18] V. Loriot, E. Hertz, B. Lavorel, and O. Faucher, J. Chem. Phys. 132, 184303 (2010).

[19] V. Renard, M. Renard, S. Guerin, Y. T. Pashayan, B. Lavorel, O. Faucher, and H. R. Jauslin, Phys. Rev. Lett. 90, 153601 (2003).

[20] V. Loriot, P. Tzallas, E. P. Benis, E. Hertz, B. Lavorel, D. Charalambidis, and O. Faucher, J. Phys. B. 40, 2503 (2007).

[21] S. Bock, E. Bich, and E. Vogel, Chem. Phys. 257, 147 (2000).

[22] A. Predoi-Cross, W. Liu, R. Murphy, C. Povey, R. R.
Gamache, A. L. Laraia, A. R. W. McKellar, D. R. Hurtmans, and V. M. Devi, JQSRT 111, 1065 (2010).

[23] U. Steinitz, Y. Prior, and I. S. Averbukh, Phys. Rev. Lett. 109, 033001 (2012).

[24] A. S. Meijer, A. S. de Wijn, M. F. E. Peters, N. J. Dam, and W. van de Water, J. Chem. Phys. 133, 164315 (2010).

[25] S. Bock, E. Bich, E. Vogel, A. S. Dickinson, and V. Vesovic, J. Chem. Phys. 121, 4117 (2004).

\section{Acknowledgments}

This work was supported by the Conseil Régional de Bourgogne, the ANR COMOC, and the FASTQUAST ITN Program of the 7th FP. 